\title{
Effect of hydroxychloroquine in patients with $\lg A$ nephropathy with insufficient responses to immunosuppressive therapy: a retrospective case-control study
}

\author{
Chen Tang, Ji-Cheng Lv, Su-Fang Shi, Yu-Qing Chen, Li-Jun Liu* and Hong Zhang
}

\begin{abstract}
Background: Hydroxychloroquine (HCQ) is a well-known immunomodulator that was recently used in immunoglobulin A (IgA) nephropathy (IgAN) due to its antiproteinuric effects. We investigated the effects of HCQ in patients with IgAN whose proteinuria remained above $1 \mathrm{~g} / \mathrm{d}$ after conventional immunosuppressive (IS) therapy.

Methods: This study was a retrospective case-control study. Twenty-six patients with IgAN who received HCQ and had insufficient responses to IS therapy (corticosteroid (CS) therapy with/without IS agents) were included. Twentysix matched historical controls who received conventional IS therapy were selected using propensity score matching. The clinical data from 6 months were compared.

Results: Proteinuria at baseline was comparable between the "IS therapy plus HCQ" and "conventional IS therapy" groups (2.35 [interquartile range (IQR), 1.47, 2.98] vs. 2.35 [IQR, 1.54, 2.98] g/d, $p=0.920)$. A significant reduction in proteinuria was noted in IgAN patients with HCQ treatment $(2.35$ [IQR, 1.47, 2.98] vs. $1.10[\mathrm{IQR}, 0.85,1.61] \mathrm{g} / \mathrm{d}, p=0.002)$. The percent reduction in proteinuria at 6 months was similar between the two groups $(-39.81 \%[-66.26,-12.37]$ vs. $-31.99 \%[-67.08,-9.14], p=0.968)$. The cumulative frequency of patients with a $50 \%$ reduction in proteinuria during the study was also comparable between the two groups (53.8\% vs. $57.7 \%, p=0.780$ ). No serious adverse events (SAEs) were observed during the study.
\end{abstract}

Conclusions: Use of HCQ achieved has similar reduction in proteinuria compared to conventional IS therapy in patients with IgAN who had insufficient responses to IS therapy.

Keywords: IgA nephropathy, Hydroxychloroquine, Immunosuppressive therapy, Proteinuria

\footnotetext{
*Correspondence: lijun.liu@aliyun.com

Renal Division, Peking University First Hospital, Peking University Institute of Nephrology, Key Laboratory of Renal Disease, Ministry of Health of China, Key Laboratory of Chronic Kidney Disease Prevention and Treatment (Peking

University), Ministry of Education, Beijing 100034, People's Republic of China
}

(c) The Author(s). 2020 Open Access This article is licensed under a Creative Commons Attribution 4.0 International License, which permits use, sharing, adaptation, distribution and reproduction in any medium or format, as long as you give appropriate credit to the original author(s) and the source, provide a link to the Creative Commons licence, and indicate if changes were made. The images or other third party material in this article are included in the article's Creative Commons licence, unless indicated otherwise in a credit line to the material. If material is not included in the article's Creative Commons licence and your intended use is not permitted by statutory regulation or exceeds the permitted use, you will need to obtain permission directly from the copyright holder. To view a copy of this licence, visit http://creativecommons.org/licenses/by/4.0/. The Creative Commons Public Domain Dedication waiver (http://creativecommons.org/publicdomain/zero/1.0/) applies to the data made available in this article, unless otherwise stated in a credit line to the data. 


\section{Background}

Immunoglobulin A (IgA) nephropathy (IgAN) is the most common primary glomerular disease worldwide, and it is a leading cause of end-stage kidney disease (ESKD) [1]. IgAN is a complex disease with variable clinical and pathological features and a partially understood pathogenesis. Therefore, no specific therapies targeting the key pathways involved in its pathogenesis are available [2]. Under these circumstances, blood pressure optimization and renin-angiotensin-aldosterone system inhibitors (RAASis) are the primary management methods [3]. The 2012 Kidney Disease Improving Global Outcomes (KDIGO) Clinical Practice Guidelines for glomerulonephritis suggest that patients with persistent proteinuria ( $\geq 1 \mathrm{~g} / \mathrm{d}$ and an estimated glomerular filtration rate $[\mathrm{eGFR}]>50 \mathrm{ml} / \mathrm{min}$ per $1.73 \mathrm{~m}^{2}$ ) after $3-6$ months of optimized supportive care should receive a 6-month course of corticosteroid (CS) therapy [4]. However, the effect of CS in IgAN is controversial. Although the STOPIgAN and TESTING studies showed that treatment with CS in IgAN led to a reduction in proteinuria, treatment with CS was associated with a significantly increased risk of serious adverse events (SAEs) [5]. Several clinical trials examined whether combination therapy with steroids and immunosuppressive (IS) agents, such as cyclophosphamide (CTX) [6], mycophenolate mofetil [7], tacrolimus [8], and leflunomide [9], produced better renal outcomes. However, the results were inconclusive [10].

Hydroxychloroquine (HCQ) is a widely used medication for the treatment of rheumatoid arthritis and systemic lupus erythematosus, and a number of experimental and clinical observations confirmed its beneficial effects [11]. Our previous studies showed that the application of HCQ in patients with IgAN effectively reduced proteinuria. Compared with conventional RAASi treatment alone, HCQ more effectively reduced proteinuria after 6 months $[12,13]$. Compared with CS therapy, HCQ was only slightly less effective for reducing proteinuria and had fewer side effects [14]. It may be effective in treating IgAN through suppressing Toll-like receptor (TLR) stimulation and reduced production of cytokines [15]. Therefore, HCQ might be an alternative or supplement to CS therapy for patients with IgAN who have insufficient responses to conventional IS therapy. Therefore, in this study, we aimed to compare the efficacy and safety of HCQ to those of conventional IS therapy in such patients.

\section{Methods}

\section{Study design and study population}

This study was a retrospective case-control study performed at a single centre. We retrospectively searched the medical records from an IgAN database at Peking University First Hospital. This database contained 1360 patients from 1994 to 2019 . The inclusion criteria were patients with IgAN who had insufficient responses to IS therapy (CS therapy with/without IS agents) and were treated with HCQ. Insufficient response to IS therapy in IgAN patients was defined as persistent proteinuria above $1 \mathrm{~g} / \mathrm{d}$ after at least 3 months of IS therapy. The exclusion criteria were a lack of baseline or follow-up data, less than 3 months of HCQ therapy, and the use of other IS agents within 3 months before or after HCQ therapy.

For each patient who received HCQ therapy, we selected matched controls from patients who received conventional IS agents in combination with CS therapy using propensity score matching based on age, sex, eGFR and proteinuria levels. A 1:1 fashion control group with the closest propensity score (within 0.2 standard deviations) for each HCQ user was selected, excluding HCQ users without a suitable match and the rest of controls. The number of matches determined the size of sample. Patients with crescentic IgAN (defined by crescents in greater than $50 \%$ of glomeruli), minimal renal disease changes with IgA deposits, acute or subacute tubulointerstitial nephritis, nephrotic syndrome (proteinuria level $\geq 3.5 \mathrm{~g} / \mathrm{d}$ and serum albumin $\leq 30 \mathrm{~g} / \mathrm{l}$ ), a greater than $30 \%$ decline in eGFR in the previous 6 months, acute kidney injury, and malignant hypertension were excluded from both groups [14] (Fig. 1).

\section{Interventions}

The HCQ dose varied according to the baseline eGFR. The dose was $0.2 \mathrm{~g}$ twice daily for patients with an eGFR greater than $60 \mathrm{ml} / \mathrm{min} / 1.73 \mathrm{~m}^{2}$, and the dose was $0.1 \mathrm{~g}$ two or three times daily for patients with an eGFR between 30 and $60 \mathrm{ml} / \mathrm{min} / 1.73 \mathrm{~m}^{2}$. However, the dose was $0.1 \mathrm{~g}$ once daily for patients with an eGFR between 15 and $30 \mathrm{ml} / \mathrm{min} / 1.73 \mathrm{~m}^{2}$ [13]. All patients were given sufficient angiotensin-converting enzyme inhibitors (ACEIs)/angiotensin receptor blockers (ARBs) for at least 3 months. The dose of RAASis was titrated to ensure that the patients were receiving the maximum allowable or tolerated dose, and blood pressure control was optimized according to the KDIGO guidelines for IgAN. Additional antihypertensive medications were prescribed if the blood pressure was $>130 / 80 \mathrm{mmHg}$ with RAASi treatment alone.

CS treatment included oral steroids $(0.8-1.0 \mathrm{mg} / \mathrm{kg} / \mathrm{d}$, maximum $60 \mathrm{mg} / \mathrm{d}$ ) for 2 months. This therapy was reduced by $5 \mathrm{mg}$ every 2 weeks and stopped within 6 to 8 months. The other was intravenous steroid (methylprednisolone $(500 \mathrm{mg}$ ) for 3 days at 1, 3 and 5 months followed by prednisone (15 mg/d p.o.) for 6 months) [14].

Other IS agent doses were standard doses based on patient renal function and physician experience. The IS agents used included CTX, mycophenolate mofetil, cyclosporine A (CsA), leflunomide and FK506. 


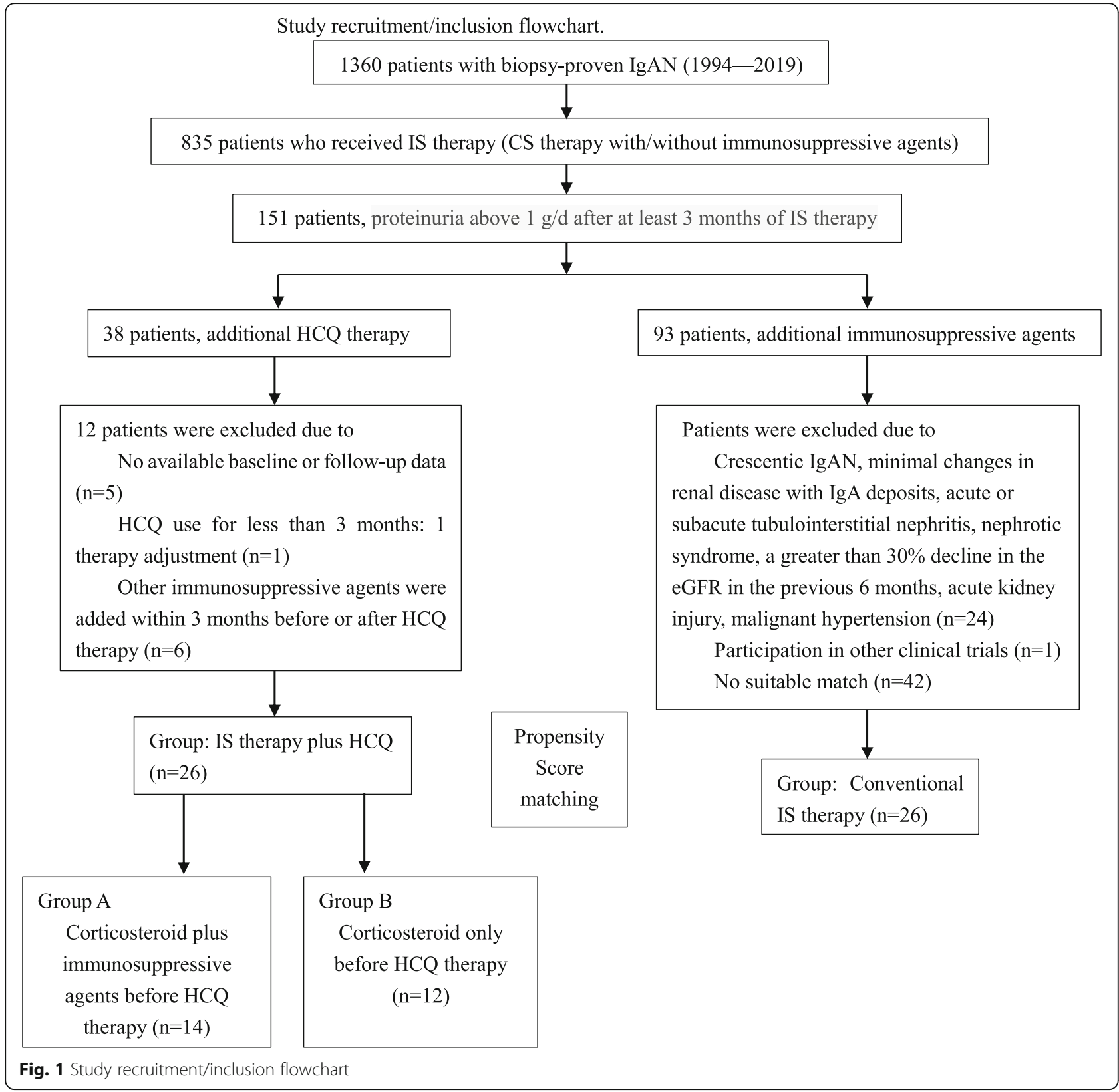

Observation lasted for 6 months or was stopped until termination of HCQ treatment or the addition of other IS agents.

\section{Data collection and outcome measures}

Baseline characteristics were reviewed for the following data: age, gender, Oxford Classification MESTC score, the duration of illness to the initiation of IS therapy, concomitant use of an RAASi, 24-h total urinary protein excretion, serum creatinine level (SCr), and eGFR. The eGFR was calculated using the Chronic Kidney Disease Epidemiology Collaboration (CKD-EPI) formula and SCr. These data were collected every 1-2 months during the follow-up. All assays were performed at a laboratory in the Department of Clinical Laboratory of Peking First Hospital using standard methods.

The primary outcome was defined as a change in proteinuria from baseline to 6 months. The secondary outcomes were defined as the percent changes in proteinuria from baseline to 2 and 4 months, the cumulative frequency of patients with a $50 \%$ decrease in proteinuria, a change in eGFR during the follow-up and adverse events (AEs) [14].

SAEs were defined as any medical events that met one or more of the following criteria: resulted in death, was life-threatening, required inpatient hospitalization or prolongation of an existing hospitalization, resulted in 
persistent or significant disability, severe infection requiring hospitalization, osteonecrosis or bone fracture, gastrointestinal haemorrhage or perforation, new-onset diabetes mellitus, new-onset cataract or fundus lesions, severe liver dysfunction or allergies requiring hospitalization, and major cardiocerebral vascular disease (including fatal/ nonfatal myocardial infarction, fatal/nonfatal stroke, and heart failure) [16].

AEs were collected from medical records. Patients receiving HCQ treatment were referred to an ophthalmologist for retinal evaluations every $3-6$ months.

\section{Statistical analysis}

Normally distributed data are presented as the means \pm $\mathrm{SD}$, and non-normally distributed data are presented as medians (Q25, Q75). Categorical data are summarized as counts and percentages. Clinical parameters during the follow-up period were compared using Student's ttests (for normally distributed, continuous variables), Wilcoxon signed-rank tests (for nonnormally distributed, continuous variables) or $\chi^{2}$ tests (for nominal variables), as appropriate. The cumulative frequency of patients with a $50 \%$ decrease in proteinuria was estimated using the Kaplan-Meier method, and time represented the period from baseline to the first occurrence of a $50 \%$ decrease in proteinuria. Univariable regression followed by multivariable logistic regression was performed to determine the independent predictors of a proteinuria reduction of at least $50 \%$.

All missing information was treated as missing data without imputation. All analyses were performed using SPSS Statistics, version 22.0 (SPSS Inc., Chicago, IL, USA). A $p$-value less than 0.05 was considered statistically significant.

\section{Results}

\section{Baseline characteristics}

A total of 26 eligible patients with IgAN from 1994 to Dec 2019 were included in this study. All patients received HCQ therapy after at least 3 months of CS therapy with/without IS agents, and no patients were treated with other immunosuppressants after receiving HCQ therapy. On average, they received IS therapy for 7.4 (4.9, 10.8) months before taking HCQ. Two of the 26 patients stopped CS use when HCQ treatment was initiated, and the remaining patients continued to use CS plus HCQ for $3.38(1.81,7.64)$ months.

Among the 26 patients treated with HCQ, 14 patients were initially treated with CS therapy plus IS agents before HCQ treatment (group A), half of whom were treated with CS plus CTX, and the average cumulative dose of cyclophosphamide was greater than $7 \mathrm{~g}$. All 14 patients in this group stopped taking IS agents when starting HCQ treatment. The other 12 patients were initially treated with CS therapy alone before HCQ treatment (group B).

Twenty-six patients with IgAN treated with conventional IS therapy were selected using propensity score matching (Fig. 1). They were initially treated with CS therapy for $3.9(2.1,11.3)$ months then treated with additional IS agents because of an insufficient response to CS therapy alone. Fourteen of these patients were treated with CTX.

The baseline characteristics of all patients are shown in Table 1. The characteristics of groups A and B are shown in Additional file 1. The baseline proteinuria levels $(2.35[1.47,2.98]$ vs. $2.35[1.54,2.98] \mathrm{g} / \mathrm{d}, p=$ $0.920)$ and eGFR $(47.65[34.65,67.48]$ vs. 51.59 [26.47, $82.32] \mathrm{ml} / \mathrm{min} / 1.73 \mathrm{~m} 2, p=0.855)$ were comparable between the IS therapy plus HCQ and conventional IS therapy groups.

\section{Primary outcome}

The level of proteinuria at 6 months decreased from 2.35 $[1.47,2.98] \mathrm{g} / \mathrm{d}$ to $1.10[0.85,1.61] \mathrm{g} / \mathrm{d}(p=0.002)$ in the IS therapy plus HCQ group and from $2.35[1.54,2.98] \mathrm{g} /$ $\mathrm{d}$ to $1.24[0.87,2.58] \mathrm{g} / \mathrm{d}(p=0.009)$ in the conventional IS therapy group. No significant difference in proteinuria at 6 months was identified between the two groups ( $p=$ 0.312 ). For groups $A$ and $B$, the level of proteinuria decreased from $2.72[1.41,3.23] \mathrm{g} / \mathrm{d}$ to $1.13[0.86,1.73] \mathrm{g} / \mathrm{d}$ $(p=0.022)$ and from $2.28[1.55,2.78] \mathrm{g} / \mathrm{d}$ to $0.90[0.81$, $1.41] \mathrm{g} / \mathrm{d}(p=0.043)$, respectively (Fig. 2$)$.

The percent reductions in proteinuria from baseline to 6 months were $-39.81 \%$ [-66.26, -12.37$]$ in the IS therapy plus HCQ group and $-31.99 \%[-67.08,-9.14]$ in the conventional IS therapy group. No significant difference in the percent change was found between the two groups $(p=0.968)$. For groups $\mathrm{A}$ and $\mathrm{B}$, the change rates were $-38.93 \%[-68.50,0.00]$ and $-44.72 \%$ [-63.30, 19.57], respectively. No significant difference in proteinuria at 6 months was observed between groups A and B $(p=0.898)$ (Fig. 3).

\section{Secondary outcomes}

The percent reductions in proteinuria from baseline to 2 months were $-40.17 \%[-48.71,-20.88]$ for patients treated with HCQ and-13.4\% [-61.66,42.97] for patients treated with conventional IS therapy $(p=0.347)$. The percent reductions in proteinuria from baseline to 4 months were $-33.62 \%[-49.15,-13.95]$ for patients treated with HCQ and $-30.43 \%[-64.4,0.00]$ for patients treated with conventional IS therapy $(p=0.768)$ (Fig. 3).

During the follow-up, the eGFR remained roughly stable. For patients in the IS therapy plus HCQ group, the eGFR was $47.65[34.65,67.48] \mathrm{ml} / \mathrm{min} / 1.73 \mathrm{~m}^{2}$ before treatment with HCQ. After 6 months of HCQ therapy, the eGFR was $50.91[30.96,71.14] \mathrm{ml} / \mathrm{min} / 1.73 \mathrm{~m}^{2}$, 
Table 1 Main clinical and laboratory characteristics at baseline in patients with IgAN

\begin{tabular}{|c|c|c|c|}
\hline & $\begin{array}{l}\text { IS therapy plus HCQ } \\
N=26\end{array}$ & $\begin{array}{l}\text { Conventional IS therapy } \\
N=26\end{array}$ & $P$-value \\
\hline \multicolumn{4}{|l|}{ Gender } \\
\hline Male/female & 9/17 & $10 / 16$ & 0.773 \\
\hline Age at renal biopsy (years) & $28.8 \pm 10.2$ & $30.8 \pm 12.2$ & 0.522 \\
\hline Duration of IS therapy before HCQ/other IS agents (months) & $7.4(4.9,10.8)$ & $3.9(2.1,11.3)$ & 0.079 \\
\hline Proteinuria level before IS therapy $(\mathrm{g} / \mathrm{d})$ & $2.52(1.66,5.60)$ & $2.27(1.54,4.36)$ & 0.727 \\
\hline Baseline proteinuria (g/d) & $2.35(1.47,2.98)$ & $2.35(1.54,2.98)$ & 0.920 \\
\hline Baseline eGFR (ml/min/1.73 m²) & $47.65(34.65,67.48)$ & $51.59(26.47,82.32)$ & 0.855 \\
\hline \multicolumn{4}{|l|}{ Oxford Classification } \\
\hline M 0/1 & $2 / 21$ & $9 / 17$ & 0.030 \\
\hline E 0/1 & $11 / 12$ & $14 / 12$ & 0.674 \\
\hline $\mathrm{S} 0 / 1$ & $7 / 16$ & $15 / 11$ & 0.056 \\
\hline T $0 / 1 / 2$ & $3 / 18 / 2$ & $11 / 11 / 4$ & 0.034 \\
\hline $\mathrm{C} 0 / 1 / 2$ & $5 / 15 / 3$ & 10/16/0 & 0.104 \\
\hline RAASi therapy & 25 & 22 & \\
\hline ACEI alone & 14 & 4 & \\
\hline ARB alone & 11 & 6 & \\
\hline ACEI plus ARB & 0 & 12 & \\
\hline Use of statins & 6 & 8 & \\
\hline \multicolumn{4}{|l|}{ Corticosteroid treatment } \\
\hline Corticosteroid pulse therapy & 13 & 8 & \\
\hline Oral corticosteroids & 13 & 18 & \\
\hline Immunosuppressive agents & $14 / 26$ & $26 / 26$ & \\
\hline Cyclophosphamide & 6 & 14 & \\
\hline Mycophenolate mofetil & 3 & 3 & \\
\hline Cyclosporine A & 2 & 4 & \\
\hline Leflunomide & 2 & 4 & \\
\hline FK506 & 1 & 1 & \\
\hline
\end{tabular}

The histological scores of 3 patients in the group "IS therapy plus HCQ" were unavailable because they underwent renal biopsy in other clinics

which was slightly higher than the beginning of treatment $(p=0.005)$. For patients treated with conventional IS therapy, no significant change in the eGFR was noted $(51.59[26.47,82.32]$ vs. $56.39[19.37,79.24] \mathrm{ml} / \mathrm{min} /$ $\left.1.73 \mathrm{~m}^{2}, p=0.305\right)$. No significant difference in the eGFR at 6 months was found between the two groups (50.91 [30.96, 71.14] vs. $51.59[26.47,82.32] \mathrm{ml} / \mathrm{min} / 1.73 \mathrm{~m}^{2}$, $p=0.821$ ) (Fig. 4).

The cumulative frequencies of patients with a $50 \%$ decrease in proteinuria at 6 months were $53.8 \%$ for patients treated with HCQ and $57.7 \%$ for patients treated with conventional IS therapy. No significant difference $(p=0.780)$ was observed between the two groups (Fig. 5).

Multivariable analysis with logistic regression did not show a specific predictive factor for a greater effective proteinuria reduction during therapy (Table 2).

\section{Safety and AEs}

No SAEs were recorded during the 6 months of observation. One patient in group A stopped HCQ therapy because of nausea and diarrhoea, and one patient had skin pigmentation without changes in medications. No AEs were recorded in group B. One patient in the conventional IS therapy group stopped CsA because of papules, and one patient stopped CTX because of pregnancy.

\section{Discussion}

The current study investigated the efficacy of HCQ after IS therapy for proteinuria in patients with IgAN and found a similar effect as conventional IS therapy in matched historical controls. All of the patients with IgAN underwent medication adjustments because of insufficient responses to initial IS or CS therapy. The protein excretion of these patients remained above $1 \mathrm{~g} / \mathrm{d}$ 


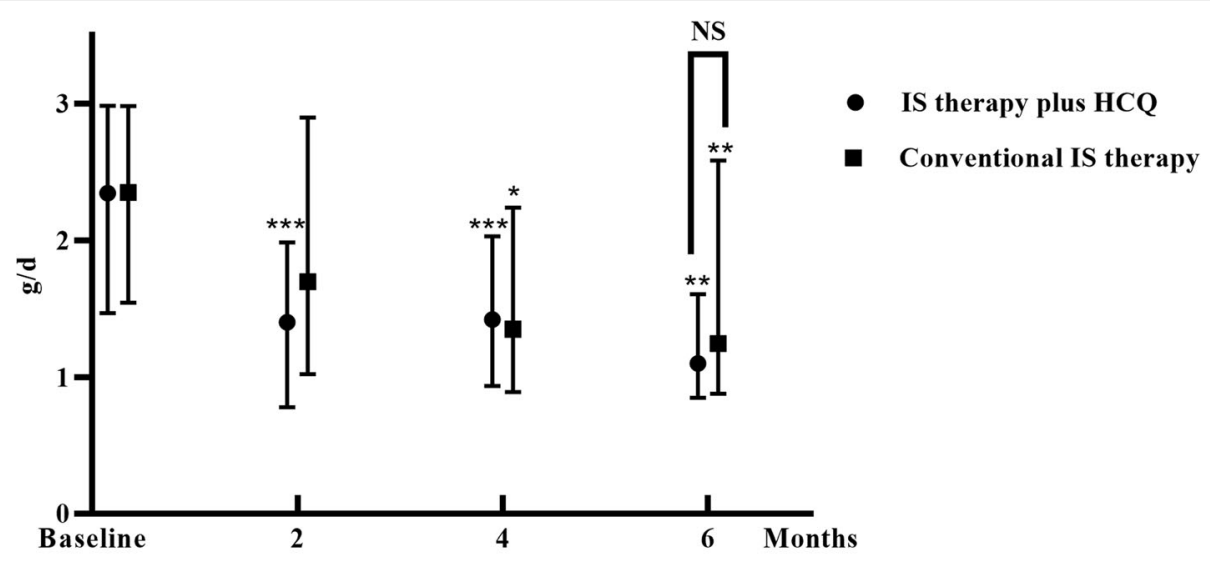

A

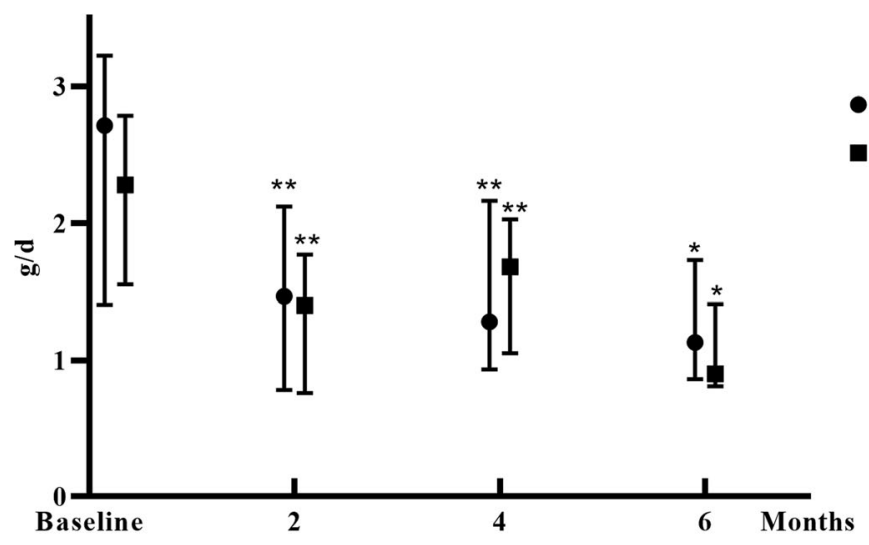

B

Fig. 2 Urinary protein excretion of all patients during the follow-up period. The dots represent the median proteinuria values, and the bars represent the 25th and 75th percentiles. The level of UTP was compared to the baseline level every 2 months. ${ }^{*} p<0.05 ;{ }^{* *} p<0.01 ;{ }^{* * *} p<0.001$. a. The groups "IS therapy plus HCQ" and "conventional IS therapy". b. Groups A and B. Group A: Patients treated with corticosteroid plus immunosuppressive agents before HCQ therapy. Group B: Patients treated with corticosteroid only before HCQ therapy

after at least 3 months of IS therapy (CS therapy with/ without IS agents). After these adjustments, the proteinuria levels in both groups decreased further at 6 months, and no significant difference in the percent change in proteinuria was noted between the two groups. HCQ may be effective in combination with other immunosuppressants for slowing disease progression, which suggests that HCQ is an alternative or supplement for patients with IgAN who have an insufficient response to IS therapy in the future.

Previous studies confirmed the antiproteinuric and immunomodulatory effects of HCQ in patients with IgAN in a short follow-up period, and patients showed good tolerance and compliance with HCQ therapy [13]. However, these studies focused on the effect of HCQ as an additional agent to RAASis and excluded patients who had recently taken CS or IS agents [12, 14]. Compared to previous studies, the current study focused on patients whose protein excretion could not be maintained below $1 \mathrm{~g} / \mathrm{d}$ even with adequate IS treatment. We found that most patients started HCQ near the end stage of IS therapy, which may guarantee that HCQ effectively reduced proteinuria and minimized interference from pretreatment immunosuppression as much as possible. The patients in the current study initiated IS therapy approximately $2.9(0.1,27.2)$ months after kidney biopsy, which also increased the connection between the pathology and clinical manifestations of the disease. The patients treated with $\mathrm{HCQ}$ in this study had higher percentages of $\mathrm{T} 1$ and $\mathrm{T} 2$ disease on pathology than the patients treated with conventional IS therapy $(86.9 \%$ vs. $57.7 \%, p=0.034$ ), which generally predicted lower renal survival and a higher risk of progression to end-stage renal disease (ESRD) [17]. However, the results suggested that HCQ may be effective in patients with IgAN with relatively severe pathology even after IS therapy. 


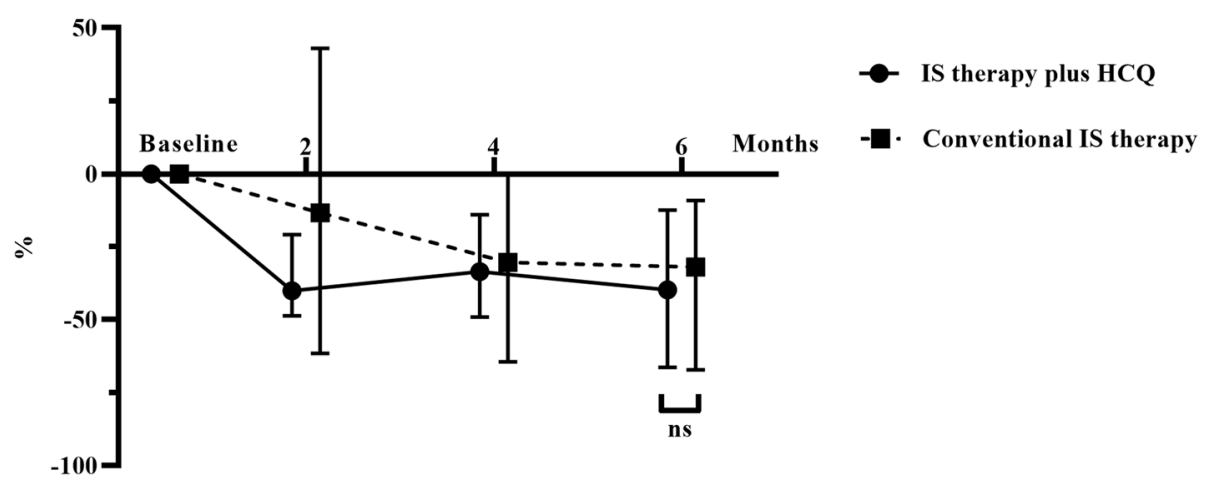

A

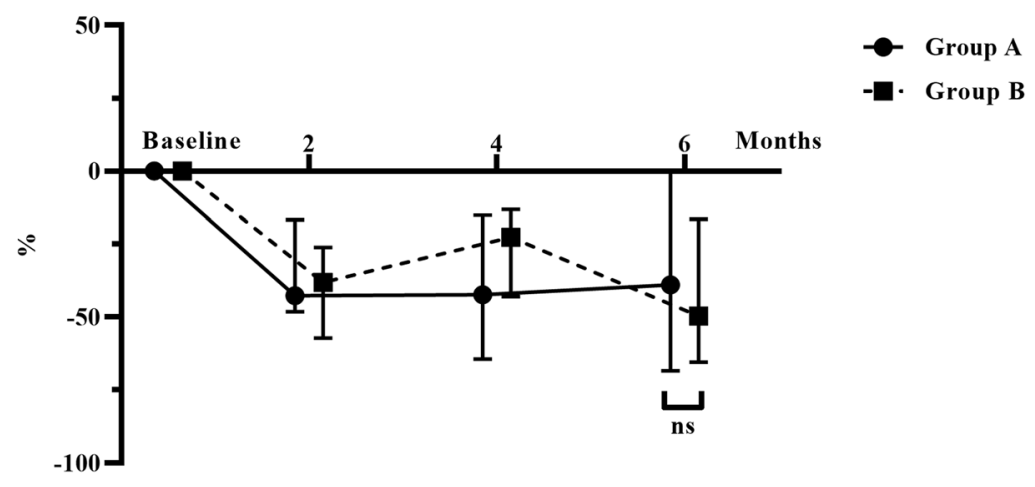

B

Fig. 3 Changes in urinary protein excretion of all patients during the follow-up period. The dots represent the median percentage changes in proteinuria, and the bars represent the 25th and 75th percentiles. The change rate was compared between two groups at 6 months. ${ }^{*} p<0.05 ;{ }^{*}$ $p<0.01$; ** $p<0.001$. a. The groups "IS therapy plus HCQ" and "conventional IS therapy". b. Groups A and B. Group A: Patients treated with corticosteroid plus immunosuppressive agents before HCQ therapy. Group B: Patients treated with corticosteroid only before HCQ therapy

Aberrant glycosylation of O-linked glycans in the hinge region of IgA1 plays a key role in the pathogenesis of IgAN [18]. However, the mechanism driving the effect of $\mathrm{HCQ}$ in reducing proteinuria and alleviating the disease in patients with IgAN was not clarified. HCQ has a considerable anti-inflammatory effect [19]. Patients in the HCQ group had proportionally higher $M$ and $E$ scores, which generally reflects glomerular inflammation [17]. IS therapy before HCQ treatment seemed effective in this study because proteinuria decreased to some degree, and the anti-inflammatory effect may have played a role. However, inflammatory factors are not the main cause of IgAN because the effect of IS therapy is not clear [20]. Recent studies suggested that TLR-mediated innate immune responses may play a role in IgAN progression. An overexpression of TLR4, TLR7, TLR8 and TLR9 is detected in patients with IgAN, which may be related to disease severity and activity [21]. HCQ blocks TLRs, such as TLRs $3,7,8$, and 9, to prevent immune system activation [19]. Complement activation by IgA1containing immune complexes is involved in the development and progression of IgA nephropathy [22], and relatively lower levels of $\mathrm{C} 3$ are associated with a greater risk of disease progression [23]. HCQ inhibited complement fixation and activation and prevented complement from splitting in vivo and in vitro [24]. These results may explain how HCQ decreased proteinuria in patients with IgAN.

The lag effects in the reduction in proteinuria in response to steroid or IS agents are noteworthy in the current study. Of the 26 patients treated with HCQ, 14 patients were treated with steroids plus IS agents (group A), and 12 patients were treated with steroids alone before HCQ therapy (group B). We did not stop immunotherapy completely for a period of time before adding $\mathrm{HCQ}$, but with the observed trend in proteinuria, the IS therapy at that time would likely not provide further benefits (Additional file 2). All patients in group A stopped IS agents when taking $\mathrm{HCQ}$, and most of them simply maintained a small dose of CS. According to the results of the TESTING study, the effect of steroids on the reduction in proteinuria was more prominent in the 


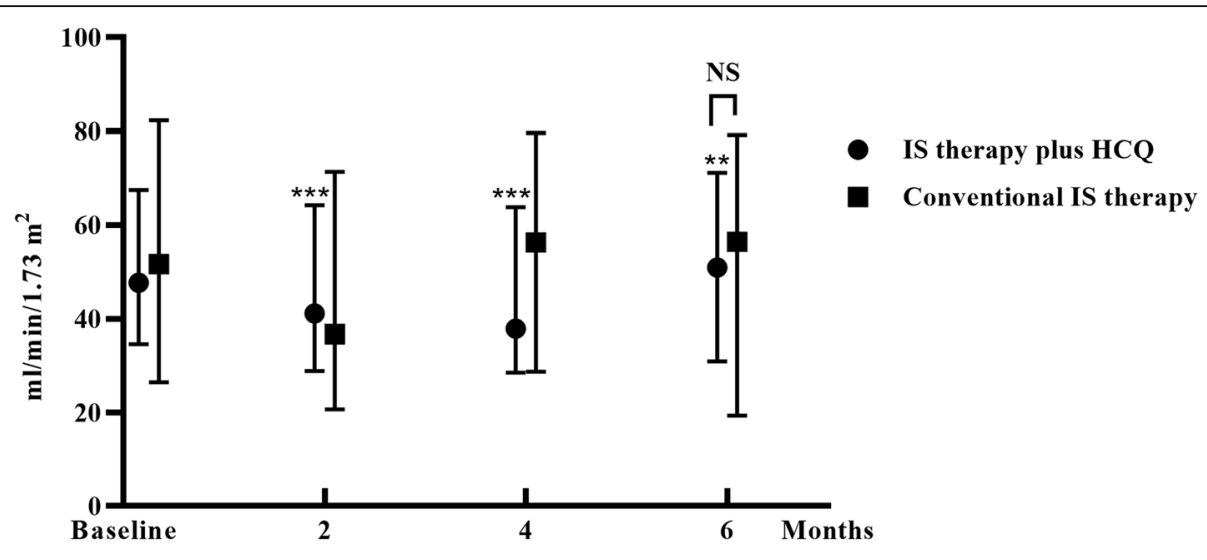

A

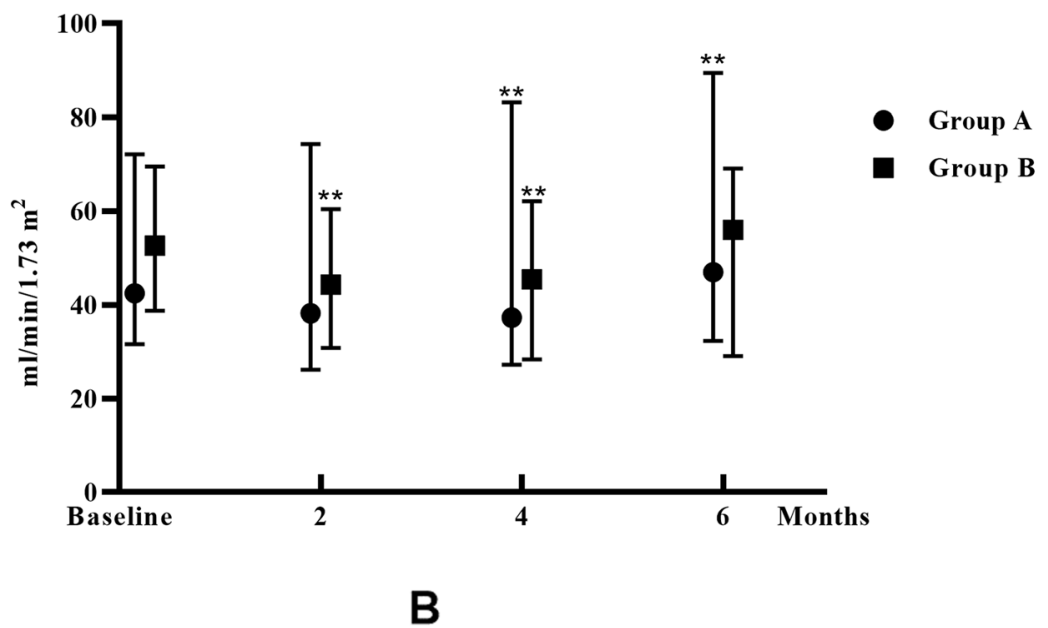

Fig. 4 The eGFR levels of all patients during the follow-up period. The dots represent the median values, and the bars represent the 25th and 75th percentiles. The eGFR was compared to the baseline eGFR every 2 months. ${ }^{*} p<0.05 ;{ }^{* *} p<0.01 ;{ }^{* * *} p<0.001$. a. The groups "IS therapy plus HCQ" and "conventional IS therapy". b. Groups A and B. Group A: Patients treated with corticosteroid plus immunosuppressive agents before HCQ therapy. Group B: Patients treated with corticosteroid only before HCQ therapy

first 3 months then gradually waned in the next 3 months [25]. The patients enrolled in our study received IS therapy for $7.4(4.9,10.8)$ months on average before taking $\mathrm{HCQ}$, which gave us more confidence that HCQ played an important role in the reduction in proteinuria during the follow-up. We also observed a relatively rapid decrease in proteinuria within 2 months after the addition of HCQ, which may be a result of the combination of CS and HCQ because most of the patients maintained low doses of CS for approximately 3 months. This speculation must be confirmed in future studies.

Multivariable analysis with logistic regression did not show a specific predictive factor for a greater effective proteinuria reduction during therapy, which may be limited by the sample size. However, HCQ is effective in combination with other immunosuppressants for slowing the deterioration of kidney function in the context of partial remission. For patients with IgAN who have insufficient responses to conventional IS therapy, HCQ may be used as an alternative or a supplement to CS therapy, rather than other additional IS agents, to minimize the side effects of CS and other IS agents.

Our study had several limitations. First, as a singlecentre retrospective study, selection bias of patients receiving steroids with or without immunosuppression was inevitable. In addition, this was a small-scale study with a short follow-up period, which may have led to some variability and bias. We didn't previously regard HCQ as an alternative treatment for patients with IgAN. In such cases, CS combined with IS treatment constituted the most suitable strategy. The long-term effect and safety of HCQ alone or HCQ plus CS in patients with IgA nephropathy and insufficient responses to IS therapy are not known. Multicentre clinical studies and mechanistic research are needed to be done in the future. 


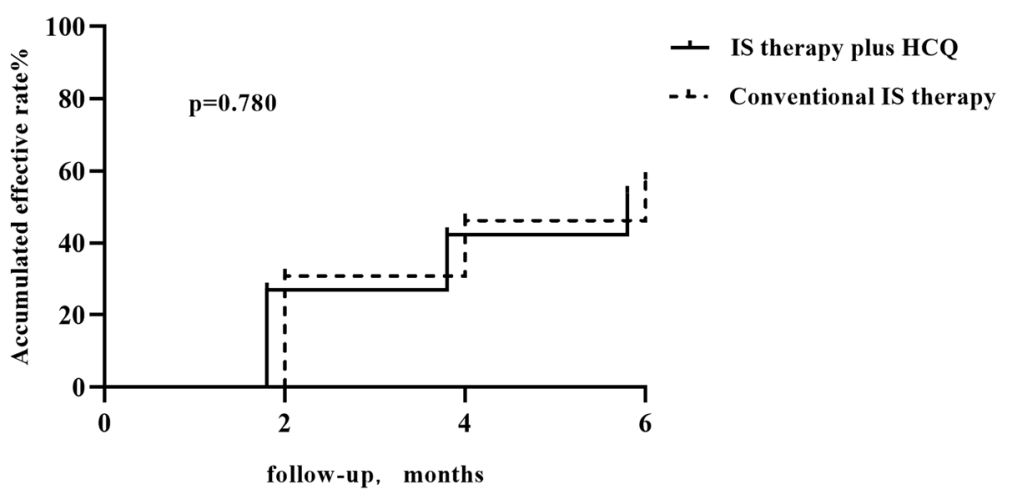

A

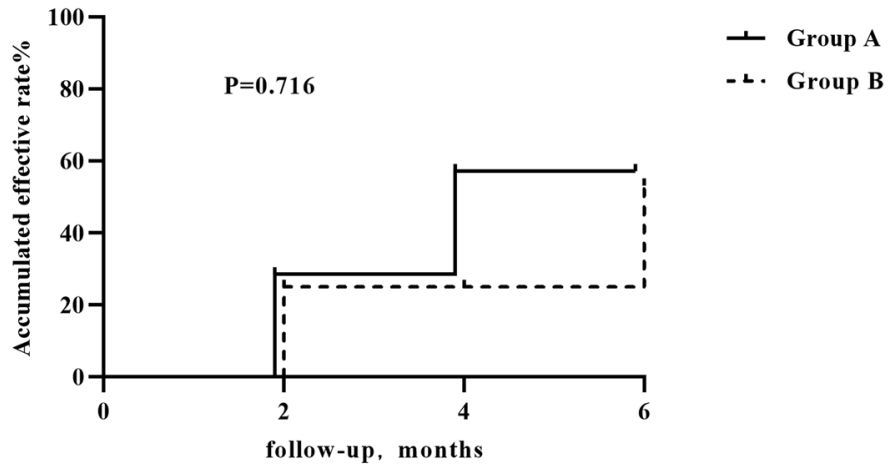

B

Fig. 5 The cumulative frequency of a 50\% reduction in proteinuria during the follow-up period. a. The accumulated effective rate for the two groups "IS therapy plus HCQ" and "conventional IS therapy". b. The accumulated effective rate for each group in the "IS therapy plus HCQ" group. Group A: Patients treated with corticosteroid plus immunosuppressive agents before HCQ therapy. Group B: Patients treated with corticosteroid only before HCQ therapy

Table 2 Clinical and histological characteristics influencing the effective proteinuria reduction frequency (a reduction in proteinuria of at least 50\%) using univariate and multivariate logistic regression

\begin{tabular}{|c|c|c|c|c|c|c|}
\hline \multirow[b]{2}{*}{ Characteristic } & \multicolumn{2}{|c|}{ Univariate } & \multirow[b]{2}{*}{$P$ value } & \multicolumn{2}{|c|}{ Multivariate } & \multirow[b]{2}{*}{$P$ value } \\
\hline & $\overline{\mathrm{OR}}$ & $95 \% \mathrm{Cl}$ & & $\overline{\mathrm{OR}}$ & $95 \% \mathrm{Cl}$ & \\
\hline Age (years) & 1.001 & $(0.952,1.053)$ & 0.961 & 1.028 & $(0.947,1.115)$ & 0.511 \\
\hline Sex (male/female) & 2.308 & $(0.747,7.128)$ & 0.146 & 1.475 & $(0.272,8.000)$ & 0.652 \\
\hline Proteinuria level before IS therapy $(\mathrm{g} / \mathrm{d})$ & 1.024 & $(0.813,1.290)$ & 0.838 & 0.883 & $(0.580,1.344)$ & 0.562 \\
\hline Baseline eGFR (ml/min/1.73 m²) & 1.009 & $(0.990,1.028)$ & 0.357 & 0.988 & $(0.960,1.017)$ & 0.415 \\
\hline Baseline proteinuria $(\mathrm{g} / \mathrm{d})$ & 1.561 & $(0.815,2.992)$ & 0.180 & 2.175 & $(0.845,3.597)$ & 0.107 \\
\hline \multicolumn{7}{|l|}{ Oxford Classification } \\
\hline $\mathrm{M} \mathrm{0/1}$ & 0.514 & $(0.129,2.052)$ & 0.346 & 0.206 & $(0.020,2.092)$ & 0.182 \\
\hline E 0/1 & 0.665 & $(0.216,2.050)$ & 0.477 & 0.478 & $(0.071,3.207)$ & 0.394 \\
\hline S 0/1 & 0.124 & $(0.034,0.452)$ & 0.232 & 0.049 & $(0.007,0.322)$ & 0.486 \\
\hline $\mathrm{T} 0 / 1 / 2$ & 0.525 & $(0.203,1.359)$ & 0.184 & 0.680 & $(0.104,4.458)$ & 0.688 \\
\hline $\mathrm{C} 0 / 1 / 2$ & 0.657 & $(0.230,1.876)$ & 0.433 & 1.039 & $(0.209,5.164)$ & 0.962 \\
\hline Optimal RAASi therapy & 0.259 & $(0.025,2.675)$ & 0.259 & 0.255 & $(0.020,3.198)$ & 0.290 \\
\hline HCQ therapy & 1.364 & $(0.457,4.071)$ & 0.578 & 0.478 & $(0.071,3.207)$ & 0.447 \\
\hline Conventional IS therapy & 0.733 & $(0.246,2.189)$ & 0.578 & 0.795 & $(0.252,2.509)$ & 0.696 \\
\hline
\end{tabular}




\section{Conclusions}

Use of HCQ achieved has similar reduction in proteinuria compared to conventional IS therapy in patients with IgAN who had insufficient responses to IS therapy. It suggests that HCQ may be an alternative treatment or a supplement for IgAN in the future to reduce the side effects of IS agents.

\section{Supplementary Information}

The online version contains supplementary material available at https://doi. org/10.1186/s12882-020-02141-9.

Additional file 1: Table S1. Main clinical and laboratory characteristics at baseline in patients treated with HCQ.

Additional file 2: Figure S1. Changes in urinary protein excretion and eGFR levels of all patients before initiation of HCQ therapy. The dots represent the median values, and the bars represent the 25th and 75th percentiles.

\section{Abbreviations}

HCQ: Hydroxychloroquine; IgAN: Immunoglobulin A nephropathy; ESKD: End-stage kidney disease; RAASi: Renin-angiotensin-aldosterone system inhibitor; CS therapy: Corticosteroid therapy; IS therapy: Immunosuppressive therapy; ACEl: Angiotensin-converting enzyme inhibitor; ARB: Angiotensin receptor blocker; CTX: Cyclophosphamide; MMF: Mycophenolate mofetil; CsA: Cyclosporine A; SAEs: Serious adverse events; AEs: Adverse events; SCr: Serum creatinine; eGFR: Estimated glomerular filtration rate (calculated using the CKD-EPI equation)

\section{Acknowledgments}

Not applicable.

\section{Authors' contributions}

Contributions: Research idea and study design: LJL. Reacher Data acquisition: $C T$, JCL, SFS, YQC, LJL and HZ. Data analysis/interpretation and statistical analysis: CT. Revise: JCL, SFS, LJL and HZ. Supervision and mentorship: JCL, SFS, YQC, LJL and HZ. All authors read and approved the final manuscript.

\section{Funding}

This study received no funding.

\section{Availability of data and materials}

The datasets used and/or analysed during the current study are available from the corresponding author upon reasonable request.

\section{Ethics approval and consent to participate}

The Peking University First Hospital Clinical Research Ethics Committee approved the study. This study was a retrospective study. All patients in the study were from our prospective IgA nephropathy cohort, in which all patients signed a consent form when they were recruited for the cohort.

\section{Consent for publication}

Not applicable.

\section{Competing interests}

The authors declare that they have no competing interests.

Received: 22 May 2020 Accepted: 30 October 2020

Published online: 10 November 2020

\section{References}

1. Rodrigues JC, Haas M, Reich HN. IgA nephropathy. Clin J Am Soc Nephrol. 2017;12(4):677-86.

2. Zhang $\mathrm{YM}$, Zhang $\mathrm{H}$. Update on treatment of immunoglobulin a nephropathy. Nephrology (Carlton). 2018;23(Suppl 4):62-7.

3. Chan TM. Corticosteroids in the treatment of IgA nephropathy: any light beyond the clouds of confusion? Postgrad Med J. 2018;94(1113):371-3.
4. Tesar V, Troyanov S, Bellur S, Verhave JC, Cook HT, Feehally J, Roberts IS, Cattran D, Coppo R. Group VsotE-EIW: corticosteroids in IgA nephropathy: a retrospective analysis from the VALIGA study. J Am Soc Nephrol. 2015;26(9):2248-58.

5. Coppo R. Treatment of IgA nephropathy: recent advances and prospects. Nephrol Ther. 2018;14(Suppl 1):S13-21.

6. Chen S, Yin Q, Ren S, Zhong X, Wang W, Li G, Wang L. A comparison of the effectiveness of cyclophosphamide, leflunomide, corticosteroids, or conservative management alone in patients with IgA nephropathy: a retrospective observational study. Sci Rep. 2018;8(1):13662.

7. Zheng JN, Bi TD, Zhu LB, Liu LL. Efficacy and safety of mycophenolate mofetil for IgA nephropathy: an updated meta-analysis of randomized controlled trials. Exp Ther Med. 2018;16(3):1882-90.

8. Wan QJ, Hu HF, He YC, Luan SD, Chen HT, Li T, Xu Y, Xu HL, Liao Y. Tacrolimus combined with low-dose corticosteroids is an effective and safe therapeutic option for refractory lgA nephropathy. Exp Ther Med. 2016;12(3):1934-8.

9. Yi J, He Z, Xu S, Feng S. Efficacy and safety of leflunomide in IgA nephropathy: a systematic review and meta-analysis. Int Urol Nephrol. 2019:51(11):1987-98.

10. Rauen T, Fitzner C, Eitner F, Sommerer C, Zeier M, Otte B, Panzer U, Peters H, Benck $U$, Mertens PR, et al. Effects of two immunosuppressive treatment protocols for IgA nephropathy. J Am Soc Nephrol. 2018;29(1):317-25.

11. Olsen NJ, Schleich MA, Karp DR. Multifaceted effects of hydroxychloroquine in human disease. Semin Arthritis Rheum. 2013:43(2):264-72.

12. Yang YZ, Liu L, Shi SF, Wang JW, Chen YQ, LV JC, Zhang H. Effects of Hydroxychloroquine on proteinuria in immunoglobulin a nephropathy. Am J Nephrol. 2018;47(3):145-52.

13. Liu LJ, Yang YZ, Shi SF, Bao YF, Yang C, Zhu SN, Sui GL, Chen YQ, Lv JC, Zhang $\mathrm{H}$. Effects of Hydroxychloroquine on proteinuria in IgA nephropathy: a randomized controlled trial. Am J Kidney Dis. 2019;74(1):15-22.

14. Yang YZ, Chen P, Liu LJ, Cai QQ, Shi SF, Chen YQ, Lv JC, Zhang $H$. Comparison of the effects of hydroxychloroquine and corticosteroid treatment on proteinuria in IgA nephropathy: a case-control study. BMC Nephrol. 2019;20(1):297.

15. Gao R, Wu W, Wen Y, Li X. Hydroxychloroquine alleviates persistent proteinuria in IgA nephropathy. Int Urol Nephrol. 2017:49(7):1233-41.

16. Cai Q, Xie X, Wang J, Shi S, Liu L, Chen Y, Lv J, Zhang H. Severe adverse effects associated with corticosteroid treatment in patients with $\lg A$ nephropathy. Kidney Int Rep. 2017:2(4):603-9.

17. Soares MF, Roberts IS. IgA nephropathy: an update. Curr Opin Nephrol Hypertens. 2017;26(3):165-71.

18. Coppo R, Fonsato V, Balegno S, Ricotti E, Loiacono E, Camilla R, Peruzzi L, Amore A, Bussolati B, Camussi G. Aberrantly glycosylated lgA1 induces mesangial cells to produce platelet-activating factor that mediates nephrin loss in cultured podocytes. Kidney Int. 2010;77(5):417-27.

19. Costedoat-Chalumeau N, Dunogue B, Morel N, Le Guern V, Guettrot-Imbert G. Hydroxychloroquine: a multifaceted treatment in lupus. Presse Med. 2014:43(6 Pt 2):e167-80.

20. Rauen T, Floege J. Inflammation in IgA nephropathy. Pediatr Nephrol. 2017; 32(12):2215-24.

21. Ciferska H, Honsova E, Lodererova A, Hruskova Z, Neprasova M, Vachek J, Suchanek M, Zima T, Coppo R, Tesar V, et al. Does the renal expression of toll-like receptors play a role in patients with IgA nephropathy? J Nephrol. 2020;33(2):307-16

22. Maillard N, Wyatt RJ, Julian BA, Kiryluk K, Gharavi A, Fremeaux-Bacchi V, Novak J. Current understanding of the role of complement in $\lg A$ nephropathy. J Am Soc Nephrol. 2015;26(7):1503-12.

23. Chen P, Yu G, Zhang X, Xie X, Wang J, Shi S, Liu L, Lv J, Zhang H. Plasma Galactose-deficient $\lg \mathrm{A} 1$ and $\mathrm{C} 3$ and CKD progression in $\lg \mathrm{A}$ nephropathy. Clin J Am Soc Nephrol. 2019;14(10):1458-65.

24. Bertolaccini ML, Contento G, Lennen R, Sanna G, Blower PJ, Ma MT, Sunassee K, Girardi G. Complement inhibition by hydroxychloroquine prevents placental and fetal brain abnormalities in antiphospholipid syndrome. J Autoimmun. 2016;75:30-8.

25. LV J, Zhang $H$, Wong MG, Jardine MJ, Hladunewich M, Jha V, Monaghan $H$, Zhao M, Barbour S, Reich H, et al. Effect of Oral methylprednisolone on clinical outcomes in patients with IgA nephropathy: the TESTING randomized clinical trial. JAMA. 2017:318(5):432-42.

\section{Publisher's Note}

Springer Nature remains neutral with regard to jurisdictional claims in published maps and institutional affiliations. 